\title{
An attribute analysis of memory: The role of the hippocampus
}

\author{
RAYMOND P. KESNER \\ University of Utah, Salt Lake City, Utah 84112
}

\begin{abstract}
A theoretical framework is proposed incorporating the multidimensional relationships between neurobiology and an attribute theory of memory representation. Within this context, a working hypothesis is proposed suggesting that the hippocampus primarily subserves the encoding, storing, and/or retrieving of both absolute spatial and long-term temporal attributes of specific episodes. Data based on electrical stimulation of the hippocampus within a number of different learning situations are presented in support of the proposed working hypothesis. In addition, data are presented to demonstrate that the hippocampus does not play a critical role in the encoding of specific episodes that encompass primarily relative spatial and/or short-term temporal attributes. Finally, data are presented to show that other neural regions might encode attributes of an episode that do not involve the encoding of absolute spatial and long-term temporal attributes.
\end{abstract}

The hippocampus has been considered as one of the primary neural regions involved in some aspect(s) of mnemonic information processing. The exact role the hippocampus plays in mnemonic function depends primarily on one's theoretical conceptions concerning the process involved in storage and retrieval of information as well as the nature of memory representation. As an example, a number of theoreticians have suggested that the hippocampus plays an important role in encoding (Butters \& Cermak, 1975), consolidation, or transfer from short- to long-term memory (Milner, 1968), or retrieval (Warrington \& Weiskrantz, 1973) of newly acquired information. Others (O'Keefe \& Nadel, 1978) have suggested that the hippocampus primarily stores information concerning space; that is, the hippocampus represents a system capable of maintaining a spatial map of the subject's geographic environment.

I would like to suggest as a working hypothesis that the hippocampus mediates the encoding, storage, or retrieval of specific attributes associated with episodes or events, that is the environmental context. Before examining this hypothesis in detail, it is first necessary to provide a background in the form of a theoretical framework and some of its underlying assumptions.

The first assumption is that long-term memory consists of a set or bundle of traces, each representing some attribute or feature of a learning experience. This assumption is not novel but, rather, has become generally accepted as a model of memory representation by many theoreticians studying human information processing (Bower, 1967; Tulving, 1972; Underwood, 1969; Wickens, 1972) and more recently in the study of animal memory (Spear, 1976). This multidimensional scaling of the memory trace contrasts with the assumption that the structure of memory consists of a monolithic trace, the strength of which can be represented by a unidimensional measure. This latter assumption is often made in interpreting results obtained within a consolidation paradigm (Gold \& McGaugh, 1975; McGaugh, 1966).

Within this multidimensional memory framework, there are at least four possibilities of how memories are stored. First, it is possible that attributes are organized in a hierarchical fashion, based upon importance. Thus, the most important attributes may be stored at one level of the hierarchy and determine the storage of less important attributes at different levels. Second, it is possible that attributes are organized independently, all attributes being of equal importance. The third possibility also suggests that attributes can be stored independently, but specific attributes can be stored at differential rates. The fourth possibility suggests that attributes are organized in a heterarchical network employing both sequential and independent systems.

The second assumption is that different neural "units" subserve different attributes. The neuroanatomical "unit" of analysis [(e.g., synapse, synaptic conglomerate, synaptic assembly, neuron, neural assembly, junctional thicket, simple circuit, neural region, system, or complex system (Welker, 1976)] cannot be specified at the present time. I will arbitrarily use neural regions such as the hippocampus, amygdala, and caudate as the initial neural units of analysis. The strong version of the assumption would propose that each specific neural region stores (contains the mnemonic representation) of a specific attribute or set of attributes. The weaker version of the assumption 
would propose that each specific neural region processes only information represented by a specific attribute or set of attributes. The organization of these attributes will largely be determined by the anatomical and functional nature of the interconnections of the critical neural regions subserving the important attributes.

Within the above mentioned organizational scheme, it becomes an important task for the researcher to identify the salient attributes of a specific experience and to select the essential neural regions that might subserve these attributes.

For example, in a typical passive avoidance training situation, the encoded memory might be composed of attributes associated with pain, environmental context (e.g., odor, illumination, location of painful shock), motivational state, affective reaction to pain (e.g., fear), and feedback from jumping and subsequent freezing responses. On the basis of experimental data, it can be postulated that the effective reaction to pain in the passive avoidance training situation might be encoded by the amygdala, the painful experience by the periaqueductal gray, the motivational state by the hypothalamus, feedback from jumping and freezing responses leading to response organization by the caudate, and the environmental context by the hippocampus. Elsewhere (Kesner, 1980), I have described detailed support for some of the suggestions mentioned above, especially the amygdala and periaqueductal gray. In this paper, I shall concentrate primarily on the hippocampus. I would like to propose that the hippocampus plays an important role in the encoding into long-term memory of specific temporal-spatial attributes of episodes associated with newly acquired information and/or the activation of specific mnemonic representations of an episode associated with previously stored information.

Knowledge of when and where episodes have occurred requires the storage and retrieval of temporarily dated events within specific places. Thus, episodes contain, in addition to specific content, both spatial and temporal attributes, that is, the environmental context.

A spatial attribute within this framework involves the encoding of specific stimuli representing places or relationships between places which can be dependent or independent of the subject's own body schema. Borrowing from distinctions made by O'Keefe and Nadel (1978), I propose that mnemonic representation of space is encoded in the form of spatial attributes by at least two different systems. One system involves the encoding of personal, relative, or egocentric spatial attributes and depends upon accurate assessment of one's body orientation in space. It is exemplified by the ability to encode and remember right-left responses. The other system involves the encoding of extra- personal, absolute, or nonegocentric spatial attributes and depends upon making discriminations with respect to spatial relationships among external stimuli. It is exemplified by the ability to encode and remember maps and localize stimuli in external space. A person who is unable to encode or retrieve both personal and extrapersonal spatial attributes of specific situations would have a loss of "topographic memory"; that is, he would be unable to orient himself in space, follow or identify routes or recognize familiar places, or use spatial cues to guide his movement from one place to another.

Furthermore, it is possible that these two spatial systems are encoded independently and are subserved by different neuronal substrates. In accordance with O'Keefe and Nadel (1978), the hippocampus would primarily mediate the encoding of absolute spatial attributes, while the parietal cortex would primarily mediate the encoding of relative spatial attributes.

A temporal attribute within this framework involves the encoding of specific stimuli or sets of spatially or temporally separated stimuli as part of an episode marking or tagging its occurrence in time, that is, separating one specific episode from previous or succeeding episodes. Borrowing from distinctions made by Atkinson and Shiffrin (1968) and Kesner (1973), I propose that mnemonic representation of an event occurring at some specified point in time is encoded in the form of temporal attributes by at least two different systems. One involves the encoding of a short-term temporal attribute and depends upon the maintenance of activated neural activity for a matter of seconds (perhaps up to a few minutes). It is exemplified by the ability to retain information for short periods of time, as measured at short retention delays (seconds). The other system involves the encoding of a long-term temporal attribute and depends upon the elaboration, growth, or consolidation of activated neural traces leading to persistence of information from seconds, to hours, to days, to a lifetime. It is exemplified by the ability to retain information for long periods of time, as measured at long retention delays (minutes, hours, days).

In accordance with a previous suggestion (Kesner, 1973), I would like to propose that these two systems can operate independently and are subserved by different neural structures, with the hippocampus primarily subserving the encoding of long-term temporal attributes and the midbrain reticular formation and frontal cortex primarily subserving the encoding of short-term temporal attributes of a situation. Thus, I am proposing that the hippocampus is primarily involved in the encoding of long-term temporal and absolute spatial attributes of episodes associated with specific learning situations. To the extent that encoded memories can be retrieved without the activation of long-term temporal and absolute spatial attributes 
(i.e., independent of specific environmental context) as exemplified by one's general knowledge of the world, the hippocampus should not play a role.

In order to test the hypothesis that the hippocampus subserves the encoding of both absolute spatial and long-term temporal attributes of a learning experience, I have primarily employed the electrical brain-stimulation (EBS) technique.

I would like to point to some important advantages of the EBS technique, especially in the context of studying the neurobiology of memory. First, the direct action of EBS appears to be reversible. Second, the onset and possibly duration of EBS action is under experimental control, which is of real importance in studying specific temporal processes associated with storage and retrieval of mnemonic information. Third, the parameters of EBS are easily manipulable. Fourth, EBS can be administered to animals that are responding (e.g., moving) in a variety of situations. Fifth, it represents a technique whose mechanism of action can be analyzed. This is of importance, because it has been assumed that by understanding the mechanism of action of an amnesiainducing agent (in this case EBS), one can strengthen the understanding of processes associated with memory.

Since it is reasonable to assume that EBS affects axons, dendrites, or cell bodies in ways which never occur with naturally triggered volleys of nerve impulses, such stimulation probably disrupts ongoing neural activity within the vicinity of the stimulating electrodes through a scrambling of normal spatio-temporal firing patterns, while indirectly facilitating or inhibiting neural activity in interconnected neural structures. It is, thus, expected that any disruptive effect on mnemonic processes or memory representations is a direct function of a disturbance of ongoing neural activity at the locus of stimulation and indirectly a function of changes in excitation and inhibition in remotely activated neural systems. However, it is also possible that activation of critical neural regions remote from the stimulation site determines the alteration of mnemonic information processing, with local effects at a particular site being less important. Thus, it is assumed that electrical stimulation of the hippocampus applied after training disrupts or allows for partial encoding of both absolute spatial and long-term temporal attributes, given that these attributes are critical components of the stored long-term memory representation. However, other attributes are not affected, thus some memory representation is stored, even though weak or impoverished. During performance or retrieval of a well-learned task, hippocampal stimulation should lead to a temporary disruption in performance to the extent that both absolute spatial and long-term temporal attributes are critical components necessary for efficient retrieval.

In all the experiments I will present, all EBS treatments are applied to the hippocampus immediately after training and, unless otherwise stated, were below electrographic or behavioral seizure level, were neither reinforcing nor punishing, and did not result in elicitation or interference with motor movements.

To reiterate, one expects hippocampal involvement in learning situations requiring the encoding or retrieval of absolute spatial and long-term temporal attributes. However, in situations involving the encoding or retrieval of relative spatial and long-term temporal attributes, absolute spatial and short-term temporal attributes, and relative spatial and short-term temporal attributes, the hippocampus should not play an important role.

\section{Situations Involving Absolute Spatial and Long-Term Temporal Attributes}

The first three situations were selected for study because each requires that the subject (in this case the rat) encodes a unique event that has occurred in a specific place.

Passive avoidance learning. Implanted rats with bilateral electrodes implanted in the hippocampus and nonoperated animals were trained to barpress in a Skinner box for sugar water. After training, all animals received a 5-mA, 1-sec-duration footshock (FS) contingent upon a barpress. Four seconds later they received $5 \mathrm{sec}$ of bilateral electrical stimulation applied to the hippocampus. Implanted and unoperated control animals received no brain stimulation. All animals were tested for retention $24 \mathrm{~h}$ later. The control groups demonstrated suppression of barpressing, indicating memory for the footshock experience. In contrast, the hippocampal stimulated animals exhibited complete amnesia (no suppression of barpressing) at the 24-h retention test (see Kesner \& Conner, 1972, for further details). Similar amnesia can be obtained at a 256-sec retention test when electrical stimulation was applied $196 \mathrm{sec}$ after the FS experience (Kesner \& Conner, 1974a).

Similarly, no retention is observed at a $24-\mathrm{h}$ retention test when EBS is applied to the hippocampus immediately after training in another type of passive avoidance learning situation. In this situation, a FS is applied when a rat is licking from a tube or a cat is eating from a dish in a specific place in a duo compartment box (McDonough \& Kesner, 1971; Baker \& Kesner, Note 1).

Others have also shown that posttrial subseizurelevel stimulation of the hippocampus in rodents can produce long-term retention deficits for passive avoidance learning (Haycock, Deadwyler, Sideroff, \& McGaugh, 1973; Kapp, Gallagher, Holmquist, \& Theall, 1978; Lidsky \& Slotnick, 1970, 1971; Sideroff, Bueno, Hirsch, Weyand, \& McGaugh, 1974; Zornetzer, Chronister, \& Ross, 1973). The results are remarkably consistent across many laboratories. Controversy arises only with seizure-level stimulation of the hippocampus, with some investigators observing long- 
term retention deficits, while others do not (Kapp, Kaufman, \& Repole, 1974; Kesner \& Doty, 1968; Vardaris \& Schwartz, 1971).

It is also important to note that lesions made in the dorsal hippocampus immediately after passive avoidance training also produced a retention deficit 1 or $24 \mathrm{~h}$ later. Similar lesions made $1 \mathrm{~h}$ after training were ineffective (Glick \& Greenstein, 1973).

Appetitive learning. The same type of disruptive effect of hippocampal stimulation upon long-term retention can also be demonstrated in an appetitive learning situation. Advantage was taken of the fact that water-deprived rats when placed in a test situation with access to an empty drinking tube will find the tube quickly, but will lick it only a few times. If, on the other hand, rats are first allowed 150 licks of a drinking tube containing a sucrose solution and $24 \mathrm{~h}$ later are presented with an empty drinking tube, they will lick the tube many times within a 3-min period. Half of the animals with electrodes implanted in the hippocampus received $30 \mathrm{sec}$ of brain stimulation commencing $4 \mathrm{sec}$ after completion of a 150 -lick sucrose experience and were retested for a 3-min period on the empty tube $24 \mathrm{~h}$ after the termination of brain stimulation. The remaining half of the implanted animals underwent the same procedure but received no brain stimulation. The total number of licks taken during the 3-min retest period was used as the main index of retention of the sucrose experience. Results indicated that, relative to implanted and unoperated controls, posttrial hippocampal stimulation produced a reliable reduction in total number of licks at the 24-h test (Berman \& Kesner, 1976). In a slightly different appetitive situation, Zornetzer and Chronister (1973) showed that posttrial stimulation of the ventral hippocampus also disrupts long-term retention.

Delayed spatial matching-to-sample. In this situation, animals are first trained to perform in an Olton eight-arm radial maze. Following acquisition, reinforcement (a piece of Fruit Loop cereal) is placed in only one of the eight arms on any one day. The location of the food varied randomly from day to day. Ten seconds after finding the food, the animal returns to the center and is contained there for a variable delay period. Following the delay, the animal must return to the same arm in order to receive a second reinforcement. The task shares many of the features described by the Maier (1932) three-table "reasoning" task, in which rats were first allowed to explore three unbaited tables, then were fed on one of the tables, and finally, were asked to choose between the baited and unbaited tables. The task is also similar to that described by Sinnamon, Freniere, and Kootz (1978), who showed that dorsal hippocampal lesions produced a severe impairment in the performance of a fourchoice delayed spatial matching-from-sample task.

Rats can delay in this eight-choice spatial matchingto-sample task up to 20 min making only a few errors (error is defined as an entry into an arm not containing the reinforcement). The ability to retain information for a 20 -min period implies a predominant involvement of long-term temporal attributes. Thus, it was not surprising to find that hippocampal electrical stimulation disrupted performance at the 20-min retention test when the treatment was applied for $10 \mathrm{sec}$ during exposure to the correct arm (Bierley \& Kesner, Note 2). In a somewhat different study, Olton (Note 3) first trained animals to perform in the eight-arm radial maze. Each rat was allowed to make four choices and then confined to the center of the apparatus and given hippocampal seizure-after-discharge-level stimulation or no stimulation. About 5 min after onset of hippocampal stimulation, the animals were released from confinement and given free choice among the arms. Only on hippocampal stimulated trials did the animals repeat the arms selected prior to treatment (complete amnesia) without making subsequent errors. Normal retention (no errors) occurred on days when no stimulation was applied. Of interest, also, is the observation that stimulation of the amygdala was ineffective. It is important to note that subseizurelevel stimulation of the hippocampus was ineffective. However, it can be shown that subseizure hippocampal stimulation becomes effective when a confinement procedure is used. In a confinement procedure, animals are delayed for $15 \mathrm{sec}$ after selection of each arm. Even though this procedure extends the time required to maintain critical information (perhaps through coding of long-term temporal attributes), no errors ensue. However, when subseizure hippocampal stimulation is applied after the selection of the first four arms, animals make many errors in selecting the remaining four arms. Thus, it appears that when the tasks (especially the last ones described above) require the encoding of absolute spatial and long-term temporal attributes of a learning experience, hippocampal stimulation is effective in preventing efficient recall of that experience.

Further support for the notion that the hippocampus mediates or encodes critical temporal-spatial attributes of episodes can be found in studies in which investigators have studied amnesic patients with presumably hippocampal damage. For example, Kinsbourne and Wood (1975) showed that amnesic patients have impoverished memory for discrete events and actual real-life occurrences, while memory for meanings, categories, and general ideas are normal. They also demonstrated that amnesic patients can often learn a rule of specific skill while forgetting when and where they learned it. Based on the above data, they have suggested, similar to the thesis proposed in this paper, that the hippocampus plays a role in the normal functioning of specific environmental contexts. They propose that these contexts are encoded by an episodic memory system, a term coined by Tulving (1972) to connote the storage and retrieval of personally 
experienced, spatially located, and temporally dated episodes.

Additional support comes from a study by Huppert and Piercy (1976), who show that Korsakoff's (presumably amnesic) patients could not easily discriminate between pictures seen $10 \mathrm{~min}$ before and those seen $24 \mathrm{~h}$ before.

Finally, Halgren, Babb, and Crandall (1978) report that changes in unit activity could be detected only from hippocampal gyrus cells when the subject (person) attempted to recall a relatively recent episodic experience, for example, location of objects in a hospital ward.

\section{Situations Involving Relative Spatial and Long-Term Temporal Attributes}

To the extent that a learning situation requires the encoding of relative spatial attributes, even though there is a necessity to encode long-term temporal attributes, the hippocampus should not play an important role. Garcia, Hankins, and Rusiniak (1974) have suggested that taste-aversion learning and taste safety learning or recovery from neophobia (habituation to novel aspects of taste) involve the encoding of relative, rather than absolute, spatial attributes. Thus, animals with electrodes implanted in the hippocampus were given a single exposure to a novel flavor (apple juice) immediately followed by hippocampal stimulation or no stimulation. Three days later, they were tested for retention of the apple juice experience by being given a second exposure to apple juice. Recovery from neophobia was indexed by an increase in apple juice consumption on the second exposure relative to the first. There were no disruptive effects on recovery from neophobia with hippocampal stimulation. Incidentally, only midbrain reticular formation stimulation was effective in disrupting recovery from neophobia (Kesner \& Berman, 1977).

The same group of hippocampal implanted animals were sometime later given a single exposure to grape juice, followed 30 min later by an injection of apomorphine (an illness-inducing agent). One minute after the apomorphine injection, the animals received either hippocampal stimulation or no stimulation. Three days later, they were tested for retention of the grapejuice-illness experience by being given a second exposure to grape juice. Learned taste aversion was indexed by a decrease in grape juice consumption on the second exposure relative to the first. There were clearly no disruptive effects on taste aversion learning. Of importance is the observation that only amygdala stimulation was effective in disrupting taste aversion learning (Kesner \& Berman, 1977). It is of significance to note that hippocampal lesions also fail to disrupt taste aversion learning (McGowan, Hankins, \& Garcia, 1972; Miller, Elkins, Fraser, Peacock, \& Hobbs, 1975; Murphy \& Brown, 1974; Nachman \& Ashe, 1974; Thomka \& Brown, 1975). Thus, in situations that require the encoding of long-term temporal and relative spatial attributes, disruption of hippocampal function does not result in alterations in retention performance.

\section{Situations Involving Absolute Spatial and Short-Term Temporal Attributes}

To the extent that a situation requires the encoding of absolute spatial attributes and the encoding of short-term temporal attributes, either because subjects are tested at short retention time intervals (seconds) or because of the inability of subjects to retain information for very long, the hippocampus should not play an important role.

In the first situation, animals were given passive avoidance training and hippocampal stimulation treatment as described earlier, but in this case they were tested for retention of the FS experience at a 64-sec delay. The same hippocampal stimulation that resulted in complete amnesia at the 24-h retention test had absolutely no disruptive effect at the short (64-sec) retention test (Kesner \& Conner, 1972, 1974a). In contrast, midbrain reticular formation stimulation disrupts retention for the FS experience at the short- but not the long-term retention test (Kesner \& Conner, 1972, 1974a). In the second situation, animals were given appetitive training and hippocampal treatment as described earlier, but in this case they were tested for retention of the 150-lick sucrose experience at a 90-sec delay. The same hippocampal stimulation that resulted in amnesia at the $24-\mathrm{h}$ retention test had no disruptive effects at the short $(90-\mathrm{sec})$ retention test (Berman \& Kesner, 1976). In the third situation, animals were given the spatial delayed matching-tosample task and hippocampal stimulation treatment as described earlier, but in this case they were tested for retention at a 1-min delay. The same stimulation that resulted in a disruption of performance at the 20-min delay had no deleterious effects at the 1-min delay (Bierley \& Kesner, Note 2).

In the fourth situation, rats were trained on a discrete-trial delayed alternation (DA) task. Training and testing were conducted in a standard operant chamber with retractable bars placed symmetrically on either side of a liquid reinforcement mechanism. On any one trial, either the right or the left bar was extended (study phase). Pressing the bar initiated its retraction and started one of five delay conditions $(0,5,15,30,40$, or $60 \mathrm{sec})$. At the end of the delay, both bars were extended (test phase) and the rat had to press the bar opposite the one it pressed last in order to receive the opportunity to drink a $.1-\mathrm{ml}$ sugar solution $(30 \% \mathrm{w} / \mathrm{v})$ reinforcement. Since no correction procedure was used, responding to either the correct or incorrect bar during the test phase initiated retraction of both bars and began a $20-\mathrm{sec}$ intertrial interval. Each daily session consisted of four 0 -sec delay warm-up trials followed by six trials at each of the five delay conditions. After 3 months 
of training according to the above procedure, the retention performance of the rats was characterized by a declining gradient across time, with chance performance at a 60-sec delay. Such a gradient has been interpreted as reflecting a process presumably associated with decay of short-term memory, and it presumably requires the operation of a short-term temporal attribute. The task also appears to require the encoding of absolute spatial attributes, since bodily orientation is an unnecessary requirement for successful performance in this task (Bierley \& Kesner, 1980).

Performance in this task remains stable from day to day, so each subject can serve as its own control. Furthermore, it becomes feasible to use multiple replications and to vary different treatment parameters (in this case, electrical brain stimulation).

After reaching a stable baseline of retention performance, animals were stereotaxically implanted with bipolar electrodes placed bilaterally in the hippocampus or midbrain reticular formation. Hippocampal stimulation at varied subseizure intensity and frequency levels was applied for $5 \mathrm{sec}$ immediately or 5 or $10 \mathrm{sec}$ after the barpress that terminated the study phase. Subseizure hippocampal stimulation was ineffective in altering delayed alternation performance. In contrast, midbrain reticular formation stimulation at low intensities was very effective in disrupting delayed alternation performance independent of locus of stimulation between the study and test phase (Bierley \& Kesner, 1980).

The lack of effect of hippocampal stimulation upon DA performance in contrast to the disruptive effect of the same level of stimulation upon delayed spatial matching-to-sample performance can be explained in the following way. When normal retention within a task is characterized by rapid forgetting, that is, information becomes unavailable within seconds due to a lack of distinctiveness of the episode, perhaps because of excessive interference or lack of novelty, presumably this task requires predominantly the operation or encoding of short-term attributes but few, if any, long-term temporal attributes. In such a situation (e.g., delayed alternation described above), hippocampal stimulation should have no effect because few, if any, long-term temporal attributes are involved in successful performance. In contrast, when normal retention within a task is characterized by relatively perfect retention for long temporal delays (minutes) due to distinctiveness of the episode, because of reduced interference or the ease of encoding long-term temporal attributes, then presumably this task requires predominantly the operation or encoding of longterm but few, if any, short-term temporal attributes.

In the latter situation (e.g., delayed spatial matchingto-sample described earlier), hippocampal stimulation should have deleterious effects, because encoding of long-term temporal attributes is presumably an important component for efficient performance.
A similar pattern of results can be found with hippocampal or fornix lesioned monkeys. They perform normally in delayed response and delayed matching-to-sample tasks in which normal monkeys show rapid forgetting (seconds) (Correll \& Scoville, 1965; Drachman \& Ommaya, 1964; Mahut, 1971; Correll \& Scoville, Note 4) but perform very poorly in a delayed matching-to-sample task in which normal monkeys show no forgetting within short (seconds) delays (Gaffan, 1976). Also, amnesic patients with presumably hippocampal damage perform normally in a Peterson short-term memory task, have a normal digit span, and show a normal "recency" effect in a free recall situation (Baddeley \& Warrington, 1970; Cermak, 1976; Starr \& Phillips, 1970). Retention in normal humans under the above conditions is characterized by rapid forgetting (Loess \& Waugh, 1967; Peterson \& Peterson, 1959). In contrast, amnesic patients show poor retention in delayed matching-tosample and delayed-paired-comparison situations, but only if, in these tasks, normal humans show good retention for long delays (minutes) (Prisko, 1963; Sidman, Stoddard, \& Mohr, 1968).

Thus, in situations in which the encoding of absolute spatial attributes might be essential but which do not require active operation of a long-term temporal attribute (at least at the time of testing), the hippocampus does not appear to play an important role.

\section{Situations Involving Relative Spatial and Short-Term Temporal Attributes}

To my knowledge, hippocampal stimulation studies using learning situations that incorporate primarily relative spatial and short-term temporal attributes have not been carried out. I am currently testing the prediction that the hippocampus does not play an important role in those types of situations.

\section{Other Attributes}

To the extent that the hippocampus encodes only long-term temporal and absolute spatial attributes of specific episodes, it should be the case that other attributes are encoded, presumably by other neural regions. Thus, in the case in which posttrial hippocampal electrical stimulation is applied after training, a subset of critical attributes would be encoded by other neural regions, in addition to some partial encoding of absolute spatial and long-term temporal attributes prior to onset of the treatment, rendering the total memory as weak, impoverished, or incomplete. In order to test the idea that some partial or qualitatively different memory is encoded following hippocampal stimulation, one can take advantage of the observation that reminder cues often serve to activate or to add to an incomplete memory, resulting in a more complete memory representation of the original learning situation (Cherkin, 1972; Gold, Haycock, Macri, \& McGaugh, 1973; Gold \& King, 1974; Kesner 
Table 1

Mean 10-Lick Latency (in Seconds) for Specific Treatment Groups as a Function of a Reminder Cue Experience Between Retention Tests 1 and 2

\begin{tabular}{lrrr}
\hline & & \multicolumn{2}{c}{ Retention } \\
\cline { 3 - 4 } \multicolumn{1}{c}{ Group } & \multicolumn{1}{c}{ Test } \\
\hline Nonoperated & 8 & 155 & 2 \\
Amygdala Stimulation & 11 & 20 & 268 \\
Amygdala Nonstimulation & 9 & 58 & 150 \\
Hippocampus Stimulation & 8 & 19 & 111 \\
Hippocampus Nonstimulation & 8 & 76 & 129 \\
\hline
\end{tabular}

\& Conner, 1974b; Miller \& Springer, 1972, 1973).

In the experiment to be described, posttrial electrical stimulation of the amygdala or hippocampus is applied after original learning in a one-trial passive avoidance task and is followed by a long-term retention test. Then a specific set of cues, composed of some component (attribute) of the original learning, is presented, followed by a second long-term retention test. In this case, the reminder cue will be noncontingent FS delivered in an environment that is different from the one used in original learning. Footshock is used as the reminder cue not only because other studies have used FS, but also because preliminary data from this laboratory have shown that when EBS is administered after a FS reminder, amygdala stimulation disrupts the reminder effect, while hippocampal stimulation does not. This latter observation implies that pain or affective reaction to pain per se are not encoded by the hippocampus.

To be more specific, rats with electrodes implanted in the amygdala or hippocampus were trained to enter a goalbox to lick a tube containing water. After reaching a predetermined latency criterion, rats were given a 3-sec 3-mA FS, followed 1 min later by hippocampal or amygdala stimulation. Implanted and nonoperated animals did not receive any stimulation. Retention was evaluated $24 \mathrm{~h}$ later as an increase in latency to enter the goalbox and lick the water tube at least 10 times. One hour after the retention test, all animals received a reminder cue (3-mA 3-sec FS) in a different apparatus (reminder cue apparatus), located in a different room. Twenty-three hours later, they were tested for the second time in the original apparatus for retention of the FS(s) experiences. It is important to note that a 3-mA 3-sec FS in the reminder cue apparatus does not increase latency to lick in the original apparatus, unless the animal had been shocked previously in the original apparatus. Also, placing the animal in the reminder cue apparatus without presentation of a FS results in a decrease in latency (less retention) on the second, as compared with the first, retention test. The data are shown in Table 1 and indicate that the nonoperated and the operated but nonstimulated control groups showed excellent retention, which was potentiated by a subsequent FS reminder cue. In contrast, stimulation of amygdala or hippocampus resulted in a retention deficit, which was reversed by presentation of the FS reminder cue for the hippocampusstimulated group. The FS reminder cue was ineffective in reinstating the memory for the amygdala-stimulated group (Baker \& Kesner, Note 1).

The data clearly suggest that other critical attributes (in this case probably affective attributes) can be encoded even though normal hippocampal function is disturbed and this encoded information might in special circumstances be sufficient for improved recall. Similar results have been attained by Winocur and Black (1978), who showed that hippocampal lesioned animals were deficient in retention of a passive avoidance learning experience, but that this deficit was reversed by presenting the subjects with a FS reminder prior to the retention test. These results are also consistent with the efficacy of reminder cues presented to human amnesics with presumed hippocampal damage (Weiskrantz \& Warrington, 1975). They found that amnesic patients' memory for previously presented pictures or words was generally poor unless partial information about the stimulus material or category cues was presented prior to retention testing, in which case there was a marked improvement in retention.

Also, it can be shown that amnesic patients can learn to form concepts and solve specific motor tasks (Corkin, 1968; Kinsbourne \& Wood, 1975; Milner, 1969; Starr \& Phillips, 1970), again demonstrating that some information not necessarily incorporating absolute spatial and long-term temporal attributes can be encoded and in special situations retrieved for efficient performance.

A summary of the results obtained with posttrial hippocampal stimulation is shown in Table 2 .

Table 2

Summary of Effects of Posttrial Subseizure Level Hippocampal Stimulation in a Variety of Learning Situations Involving the Encoding of Temporal-Spatial Attributes

\begin{tabular}{cll}
$\begin{array}{c}\text { Spatial } \\
\text { Attributes }\end{array}$ & \multicolumn{1}{c}{$\begin{array}{c}\text { Short-Term Temporal Attributes } \\
\text { (Retention test 5 to } 90 \mathrm{sec} \text { ) }\end{array}$} & \multicolumn{1}{c}{$\begin{array}{c}\text { Long-Term Temporal Attributes } \\
\text { (Retention test 5 min to 72 h) }\end{array}$} \\
\hline Absolute & $\begin{array}{l}\text { Passive avoidance (Normal) } \\
\text { Appetitive learning (Normal) }\end{array}$ & $\begin{array}{l}\text { Passive avoidance (Deficit) } \\
\text { Appetitive learning (Deficit) }\end{array}$ \\
& $\begin{array}{l}\text { Discrete delayed alternation learning (Normal) } \\
\text { Delayed-spatial matching-to-sample (Normal) }\end{array}$ & $\begin{array}{l}\text { Delayed-spatial matching-to-sample (Deficit) } \\
\text { Relative }\end{array}$ \\
& & $\begin{array}{l}\text { Recovery from neophobia (Normal) } \\
\text { Taste aversion learning (Normal) }\end{array}$ \\
\hline
\end{tabular}


In general, the results indicate that in situations that require the encoding, storage, and/or retrieval of both absolute spatial and long-term temporal attributes, posttrial hippocampal stimulation disrupts retention (usually measured from minutes to days). These findings support the working hypothesis that the role of the hippocampus is to encode, store, and/or retrieve absolute spatial and long-term temporal attributes of specific episodes. The fact that these retention deficits can be reversed with the use of appropriate reminder cues implies that different attributes of an episode can be encoded, probably by different neural regions.

In situations that require only long-term temporal attributes in conjunction with relative spatial attributes or only absolute spatial attributes in conjunction with short-term spatial attributes, hippocampal stimulation has no disruptive effects, suggesting that critical involvement of the hippocampus occurs only in situations in which both long-term temporal and absolute spatial attributes are critical components of the storage of information.

It should be clear that the theoretical framework presented above can only serve as a guide and that a great deal of work needs to be carried out to fill in the gaps and extend the findings.

\section{REFERENCE NOTES}

1. Baker, L. J., \& Kesner, R. P. Differential effects of $a$ reminder cue with hippocampus and amygdala stimulation induced amnesia. Paper presented at the annual meeting of the Western Psychological Association, San Francisco, 1978.

2. Bierley, R. A., \& Kesner, R. P. Unpublished observations. 1980.

3. Olton, D. S. The function of septo-hippocampal connections in spatially organized behavior. In Functions of the septohippocampal system. Ciba Foundation Symposium No. 58, 1978.

4. Correll, R. E., \& Scoville, W. B. Effects of medial temporal lesions on delayed reactions. Paper presented at the meeting of the American Psychological Association, Chicago, September 1960.

\section{REFERENCES}

Atkinson, R. C., \& Shiffrin, R. M. Human memory: A proposed system and its control processes. In K. W. Spence \& J. T. Spence (Eds.), Advances in the psychology of learning and motivation, research and theory (Vol. 2). New York: Academic Press, 1968.

BadDeley, A. D., \& Warrington, E. K. Amnesia and the distinction between long- and short-term memory. Journal of Verbal Learning and Verbal Behavior, 1970, 9, 176-189.

Berman, R. F., \& Kesner, R. P. Post-trial hippocampal, amygdaloid and lateral hypothalamic electrical stimulation: Effects upon memory of an appetitive experience. Journal of Comparative and Physiological Psychology, 1976, 90, 260-267.

Bierley, R. A., \& KeSNER, R. P. Short-term memory: The role of the midbrain reticular formation. Journal of Comparative and Physiological Psychology, 1980, in press.

Bower, G. A multicomponent theory of the memory trace. In K. W. Spence \& J. T. Spence (Eds.), The psychology of learning and motivation (Vol. 1). New York: Academic Press, 1967.

Butters, N., \& Cermak, L. Some analyses of amnesic syndromes in brain-damaged patients. In R. L. Isaacson \& K. H. Pribram
(Eds.), The hippocampus (Vol. 2). New York: Plenum Press, 1975.

Cermak, L. S. The encoding capacity of a patient with amnesia due to encephalitis. Neuropsychologia, 1976, 14, 311-326.

Cherkin, A. Retrograde amnesia in the chick: Resistance to the reminder effect. Physiology \& Behavior, 1972, 8, 949-955.

Corkin, S. Acquisition of motor skill after bilateral medial temporal lobe excision. Neuropsychologia, 1968, 6, 255-265.

Correll, R. E., \& Scoville, W. B. Performance on delayed match following lesions of medial temporal lobe structures. Journal of Comparative and Physiological Psychology, 1965, 60, 360-367.

Drachman, D. A., \& Ommaya, A. K. Memory and the hippocampal complex. Archives of Neurology, 1964, 10, 411-425.

GAFFAN, D. Recognition memory in animals. In J. Brown (Ed.), Recognition and recall. London: Wiley, 1976.

Garcia, J., Hankins, W. G., \& Rusiniak, K. W. Behavioral regulation of the milieu interne in man and rat. Science, 1974, $185,823-831$.

Glick, S. D., \& Greenstein, S. Comparative learning and memory deficits following hippocampal and caudate lesions in mice. Journal of Comparative and Physiological Psychology, 1973, 82, 188-194.

Gold, P. E., Haycock, J. W., Macri, J., \& McGaugh, J. L. Retrograde amnesia and the "reminder effect": An alternative interpretation. Science, 1973, 180, 1199-1201.

Gold, P. E., \& King, R. A. Retrograde amnesia: Storage failure versus retrieval failure. Psychological Review, 1974, 81, 465-469.

Gold, P. E., \& McGaugh, J. L. A single-trace, two-process view of memory storage processes. In D. Deutsch \& J. A Deutsch (Eds.), Short-term memory. New York: Academic Press, 1975.

Halgren, E., Babb, T. L., \& Crandall, P. H. Activity of human hippocampal formation and amygdala neurons during memory testing. Electroencephalography and Clinical Neurophysiology, 1978, 45, 585-601.

Haycock, J. W., De adwyler, S. A., Sideroff, S. I., \& McGAUGH, J. L. Retrograde amnesia and cholinergic systems in the caudate-putamen complex and dorsal hippocampus of the rat. Experimental Neurology, 1973, 41, 201-213.

Huppert, F. A., \& Piercy, M. Recognition memory in amnesic patients: Effect of temporal context and familiarity of material. Cortex, 1976, 12, 8-20.

Kapp, B. S., Gallagher, M., Holmquist, B. K., \& Theall, C. L. Retrograde amnesia and hippocampal stimulation: Dependence upon the nature of associations formed during conditioning. Behavioral Biology, 1978, 24, 1-23.

KaPP, B. S., Kaufman, T. D., \& RePOle, D. A. Hippocampal stimulation and memory: Effects of stimulation parameters and reinforcement magnitude. Physiology \& Behavior, 1974, 13, 47-56.

KeSner, R. P. A neural system analysis of memory storage and retrieval. Psychological Bulletin, 1973, 80, 177-203.

KESNER, R. P. Brain stimulation: Effects on memory. In R. F. Thompson \& J. L. McGaugh (Eds.), Neurobiology of learning and memory. New York: Plenum Press, 1980, in press.

Kesner, R. P., \& Berman, R. F. Effects of midbrain reticular formation, hippocampal and lateral hypothalamic stimulation upon recovery from neophobia and taste aversion learning. Physiology \& Behavior, 1977, 18, 763-768.

Kesner, R. P., \& Conner, H. S. Independence of short- and long-term memory: A neural system analysis. Science, 1972, 176, 432-434.

Kesner, R. P., \& Conner, H. S. Effects of electrical stimulation of limbic system and midbrain reticular formation upon short- and long-term memory. Physiology \& Behavior, 1974, 12, 5-12. (a)

Kesner, R. P., \& Conner, H. S. Cue-dependent recovery from ECS-induced amnesia: Evidence for time-dependence. Physiological Psychology, 1974, 2, 123-125. (b)

Kesner, R. P., \& Doty, R. W. Amnesia produced in cats by 
local seizure activity initiated from the amygdala. Experimental Neurology, 1968, 21, 58-68.

Kinsbourne, M., \& Wood, F. Short-term memory processes and the amnesic syndrome. In D. Deutsch \& J. A. Deutsch (Eds.), Short-term memory. New York: Academic Press, 1975.

Lidsky, A., \& Slotnick, B. M. Electrical stimulation of the hippocampus and electroconvulsive shock produce similar amnestic effects in mice. Neuropsychologia, 1970, 8, 363-369.

Lidsky, A., \& Slotnick, B. M. Effects of posttrial limbic stimulation on retention of a one-trial passive-avoidance response. Journal of Comparative and Physiological Psychology, 1971, 76, 337-348.

Loess, H., \& WAUGH, N. C. Short-term memory and inter-trial interval. Journal of Verbal Learning and Verbal Behavior, 1967, 6, 455-460.

Mанит, H. Spatial and object reversal learning in monkeys with partial temporal lobe ablations. Neuropsychologia, 1971, 9 , 409-424.

MaIER, N. R. F. Cortical destruction of the posterior part of the brain and its effect on reasoning in rats. Journal of Comparative Neurology, 1932, 56, 179-208.

McDonough, J. R., JR., \& Kesner, R. P. Amnesia produced by brief electrical stimulation of the amygdala or dorsal hippocampus in cats. Journal of Comparative and Physiological Psychology, 1971, 77, 171-178.

McGaUgh, J. L. Time-dependent processes in memory storage. Science, 1966, 153, 1351-1358.

McGowan, B. K., Hankins, W. G., \& Garcia, J. Limbic lesions and control of the internal and external environment. Behavioral Biology, 1972, 7, 841-852.

Miller, C. R., Elkins, R. L., Fraser, J., Peacock, L. J., \& Hoввs, S. H. Taste aversion and passive avoidance in rats with hippocampal lesions. Physiological Psychology, 1975, 3, 123-126.

Miller, R. R., \& Springer, A. D. Induced recovery of memory in rats following electroconvulsive shock. Physiology \& Behavior, 1972, 8, 645-651.

Miller, R. R., \& Springer, A. D. Amnesia, consolidation and retrieval. Psychological Review, 1973, 80, 69-79.

Milner, B. Preface: Material specific and generalized memory loss. Neuropsychologia, 1968, 6, 175-179.

Milner, B. Residual intellectual and memory deficits after head injury. In A. E. Walker, W. F. Cavenass, \& M. Critchley (Eds.), The late effects of head injury. Springfield, Ill: Thomas, 1969.

MurPhy, L. R., \& Brown, T. S. Hippocampal lesions and learned taste aversion. Physiological Psychology, 1974, 2, 60-64.

Nachman, M., \& Ashe, J. H. Effects of basolateral amygdala lesions on neophobia, learned taste aversions and sodium appetite in rats. Journal of Comparative and Physiological Psychology, 1974, 87, 622-643.

O'KeEFE, J., \& NADEL, L. The hippocampus as a cognitive map. Oxford: Clarendon Press, 1978.

Peterson, L. R., \& Peterson, M. T. Short-term retention of individual verbal items. Journal of Experimental Psychology, 1959, 58, 193-198.

Prisko, L. Short-term memory in cerebral damage. Unpublished $\mathrm{PhD}$ dissertation, McGill University, 1963.
Sideroff, S., Bueno, O., Hirsch, A., Weyand, T., \& McGaugh, J. Retrograde amnesia initiated by low-level stimulation of hippocampal cytoarchitectonic areas. Experimental Neurology, 1974, 43, 285-297.

Sidman, M., Stoddard, L. T., \& Mohr, J. P. Some additional quantitative observations of immediate memory in a patient with bilateral hippocampal lesions. Neuropsychologia, 1968, 6, 245-254.

Sinnamon, H. M., Freniere, S., \& Kootz, J. Rat hippocampus and memory for places of changing significance. Journal of Comparative and Physiological Psychology, 1978, 92, 142-155.

SPEAR, N. F. Retrieval of memories: A psychobiological approach. In W. K. Estes (Ed.), Handbook of learning and cognitive processes (Vol. 4), Attention and memory. Hillsdale, N.J: Erlbaum, 1976

Starr, A., \& Phillips, L. Verbal and motor memory in the amnesic syndrome. Neuropsychologia, 1970, 8, 75-88.

Tномка, M. L., \& Brown, T. S. The effect of hippocampal lesions on the development and extinction of a learned taste aversion for a novel food. Physiological Psychology, 1975, 3, 281-284.

Tulving, E. Episodic and semantic memory. In E. Tulving \& W. Donaldson (Eds.), Organization of memory. New York: Academic Press, 1972.

UNDERWOOD, B. J. Attributes of memory. Psychological Review. $1969,76,559-573$.

Vardaris, R. M., \& Schwartz, K. E. Retrograde amnesia for passive avoidance produced by stimulation of dorsal hippocampus. Physiology \& Behavior, 1971, 6, 131-135.

Warrington, E. K., \& Weiskrantz, L. An analysis of shortterm and long-term memory defects in man. In J. A. Deutsch (Ed.), The physiological basis of memory. New York: Academic Press, 1973.

Weiskrantz, L., \& Warrington, E. K. The problem of the amnesic syndrome in man and animals. In R. L. Isaacson \& K. H. Pribram (Eds.), The hippocampus (Vol. 2). New York: Plenum Press, 1975.

WELKER, W. I. Brain evaluation in mammals: A review of concepts, problems, and methods. In R. B. Masteron, M. E. Bitterman, B. Campbell, \& N. Hotton (Eds.), Evolution of brain and behavior in vertebrates. Potomac, Md: Erlbaum, 1976.

Wickens, D. P. Characteristics of word encoding. In A. W. Melton \& E. Martin (Eds.), Coding processes in human memory. Washington, D.C: V. H. Winston, 1972.

Winocur, G., \& Black, A. H. Cue-induced recall of a passive avoidance response by rats with hippocampal lesions. Physiology \& Behavior, 1978, 21, 39-44.

Zornetzer, S. F., \& Chronister, R. B. Neuroanatomical localization of memory disruption: Relationship between brain structure and learning task. Physiology \& Behavior, 1973, 10, 747-750.

Zornetzer, S. F., Chronister, R. B., \& Ross, B. The hippocampus and retrograde amnesia: Localization of some positive and negative memory disruptive sites. Behavioral Biology, 1973, 8, 507-518. 